\title{
The Performance Analysis of Full-Duplex System
}

\author{
Linjun Wu \\ College of Information Science and Engineering, Henan University of Technology, \\ Zhengzhou, 450001,China \\ ljwu@haut.edu.cn
}

Keywords: Full-duplex, OFDM, MIMO, WiFi, Nyquist Frequency.

Abstract. This paper presented a design using full-duplex wireless signal inversion adaptive cancellation. Signal inversion using a transmitter based on balanced / unbalanced design was simple (Balun). The new design, different from the previous work in support of broadband and high-power systems. In theory, this new design was not limited in bandwidth and power. In practice, we found that the signal inversion can be canceled at least $36 \mathrm{~dB}$ at $45 \mathrm{MHz}$ bandwidth. Further, the signal in the digital domain inversion cancellation of a combination of OFDM signal was 15MHz seventy-three db reduce self-interference.

\section{Introduction}

General radio stations are half duplex. In a single channel, you can send or receive, but not both. We can not send and receive information is the root of many of today's wireless open issues, such as MAC and bit rate adaptation. Full-duplex wireless has been completely rethink how to design and build the potential of wireless networks [1,2]. One possible orphanage in the price of a full-duplex wireless LED Knowledge can build such a device recently. The basic challenge is to reduce self-interference. If a node can not hear your own transmitted signal, then it will not interfere with specific information with other CAN packets sent and received.

As we all know, digital and analog technologies together, even, are not sufficient enough to eliminate interference from full-duplex [3,4]. Some analog noise cancellation technology using chip subtract from the transmission signal ("noise") from the received signal. Digital canceled, using CSMA / CN, fiber optic networks and full-duplex operation, at minus self-interference in the digital domain, the receiver base band digital signal samples. Because of these restrictions, recent work explores the position of the antenna as an additional cancellation technology. Use the fact that the separation distance between the antenna transmitting and receiving antennas naturally reduce self interference due to the signal attenuation [5,6]. However, to obtain a sufficient reduction in full-duplex separation between the antenna may be we need to unrealistic TX and RX antenna big distance. To further eliminate self interference, Choi [7]proposed an additional technique called antenna cancel [6]. Antenna canceled, when combined with other mechanisms that allow full-duplex operation.

Although promising, the basic design of the antenna has three main restrictions were lifted. First, they need three antennas: two transmit and one receive. Full-duplex throughput doubles, but three-antenna MIMO system throughput can be three, so from a performance standpoint, canceled the original antenna is unattractive. In addition, there are two far-field emission destructive interference of light produced by an empty area of the antenna. The second limitation is that "bandwidth limit" theoretical limit, prevent support broadband signals such as WiFi. Finally, Cui Designed by a third, practical limitations: it requires manual tuning. The manual tuning is sufficient proof of concept laboratory experiments show, it leaves the possibility of establishing a full-duplex system that can automatically adapt to the reality of the problem.

This article addresses all three limitations of a full-duplex radio design. We propose a new mechanism, using a signal inversion, by balancing (balanced / unbalanced) circuit is proposed to offset the balance algorithms. No bandwidth limit before canceling algorithm: it can theoretically support any bandwidth and cancel any high transmit power. Previous cancellation algorithm requires 
only two antennas, a transmission and reception. In addition, this paper presents an optimization algorithm that allows the previous cancellation algorithm based radio design quickly and accurately, and automatically adjust to full-duplex circuit cancels the original self-interference components.

Second, it is a contribution to offset the previous design implementation. It explores how the practice and theory of canceling algorithms to implement. For example, for a balanced system, in theory, we can provide a sound cancellation, which need to assume that the channel has a flat frequency response: If it is not balanced, the inverted signal may be different. These results suggest that may have challenges in large-scale production of a full-duplex radio in the future.

We evaluated the design of two ways. The first is the use of tightly controlled channel sounder verify the limits of existing circuits at the physical layer. We found that re-balancing the establishment of commodity component elimination circuit can be canceled at $45 \mathrm{MHz}$ bandwidth is $35 \mathrm{~dB}$. Combined with digital cancel, which makes full-duplex wireless cancel the self-interference $70 \mathrm{db}$. 802.11n equipment is therefore full duplex TX and RX antenna possible separation between reasonable. Second evaluation by using software-defined radio node test bed to quantify [the benefits of 13 full-duplex MAC layer link layer] counter flow design.

\section{System Design and Full Duplex}

This section provides background on the basics of radio design and full duplex. It explains the basic challenges to establish a full-duplex radio, so existing technologies, and limitations of these techniques. Figure 1 shows the basic design of modern radio receivers. We have taken these details, because the underlying data representation, decide how and when full-duplex radio signal can be canceled. We use 802.11b 1 channel as an example of a number of concepts that are running in the concrete floor. Wireless signal occupied bandwidth, frequency range to meet the requirements of 802.11b channel, for example, $802.11 \mathrm{~b} 1$ center channel is $2.412 \mathrm{ghz}$, the bandwidth of $22 \mathrm{MHz}$. It is from 2.423Ghz to 2.401ghz. Transmitting and receiving signals in this frequency range is called RF (Radio Frequency) signal. Because the digital channel in the $4.8 \mathrm{GHz}$ Nyquist frequency conversion, the base band signal in the radio frequency signal is concentrated $0 \mathrm{~Hz} .802 .11$ base band signal to the radio at a much lower speed analog-digital converter (ADC): $22 \mathrm{MHz}$ base band signal needs to reach or slightly above the Nyquist frequency to $44 \mathrm{MHz}$ running ADC. Commodity WiFi cards usually use 8bit samples, although some software radio can provide 12-bit resolution [6] .

Transmitting a data packet, the base station needs to generate a waveform and digital sampling, converts it into a base band signal and a digital to analog converter (DAC), and to a radio frequency base band modulation. It receives a data packet, converts the RF signal into a base band signal, and then digitally sampled base band ADC sample produced.

The goal is a full-duplex radio transmission and reception simultaneously. The problem is that a node only hear the signal that it wants to receive, and signal transmission. Cancel this self-interference received, a full-duplex node can theoretically decode the received signal. The cancellation is difficult, however, because the self-interference can millions to billions of times (60-90db) ratio of the received signal. For example, a transmit power of $0 \mathrm{dBm}$, about $90 \mathrm{dbm}$ need to cancel interference from nearly $95 \mathrm{~dB}$ to ensure its own transmission without interrupting the reception of terrestrial radio noise. There has been interference canceled digital and analog technology. Digital eliminate operating digital samples. If stage a full-duplex radio, one of its transmit signal amplitude good estimate at the receiving antenna, it can generate digital samples of their transmission signals and subtract them from the receipt of the samples. Digital cancellation, while helpful, is not enough in itself: In the research literature in the existing system canceled 20-25 $\mathrm{dB}[7,8]$. Its limitation is that $\mathrm{ADC}$ has a limited dynamic range: from interference strong, ADC reception signal to digital samples recovered. 


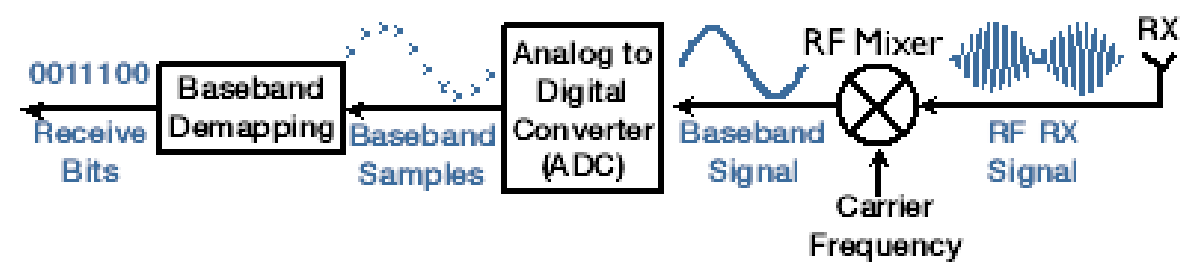

Figure 1: simplified block diagram of the RF receiver

Analog eliminate the use of the transmission of knowledge, cancel self-interference in the radio frequency signal is digitized. One method is to use a simulation to cancel the second transport chain to create self-interference [5] digital estimate analog cancellation signal to cancel 33dB self-interference in $625 \mathrm{khz}$ bandwidth signal. Another method uses a technology similar to noise-canceling headphones. Self-interference signal "noise" in qhx220 chip circuit is subtracted from the received signal. The qhx220 is $10 \mathrm{MHz}$ bandwidth signal cancel 20-25 dB but it introduces many complications, such as non-linear and distorted, complex digital eliminate obvious. We studied these restrictions in 5.1 more deeply, but the overall result is that this method does not provide more than 25 $\mathrm{dB}$ of cancellation and can not be canceled and figures: therefore insufficient for full duplex.

For the above limitations, the antenna recent study has suggested configuration technique [3,4]. Art in full duplex mode at $0 \mathrm{dBm}$ transmit power narrow band signal of $5 \mathrm{MHz}$ (MW) [4]. Designed to achieve this result the elimination of the above novel form of cancellation so-called "antenna" canceled by increasing both digital and analog. In the antenna to eliminate the key idea is to use a second transmit antenna and two transmit it so destructive interference at the receiving antenna signal. It is offset by two transmit antennas distance between a half wavelength. The resulting destructive interference cancellation 20-30db self interference. Combine analog and digital offset described above, designed to eliminate interference from 50-60 decibels, which is sufficient to operate a full-duplex 802.15.4 radio.

This design, while the breakthrough has been basically and practical limitations. The first limitation is the fundamental, to the bandwidth of the transmitted signal. Antenna canceled ensure only the center frequency of the signal is completely inverted, at the receiving antenna, thereby eliminating a whole. However, as a signal away from the center frequency, phase offset two versions from the transmitting antenna away from the perfect reversal and other two are not completely eliminated. Accordingly, the antenna performance degradation of signal cancellation increase bandwidth.

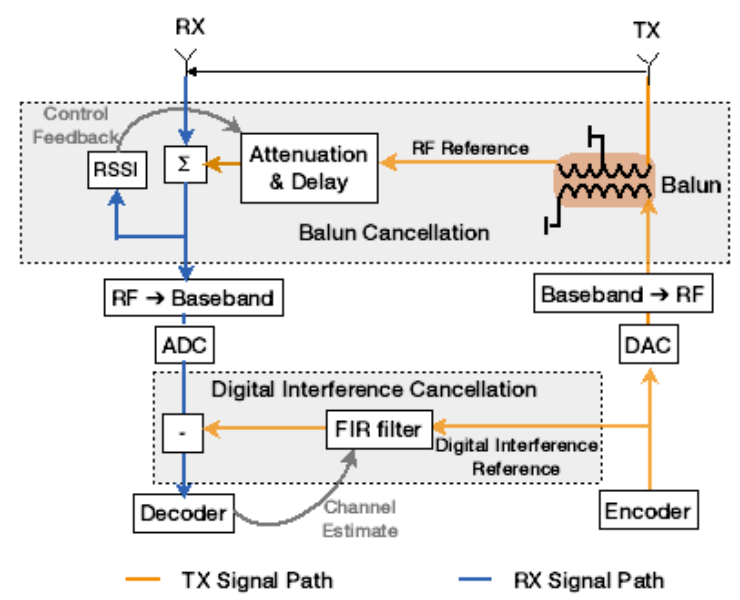

Figure 2 full system block diagram

In practice, this means that the antenna can not be canceled Cancel 802.11b signal exceeds 30dB: [7,8]. Please note, it may just be possible to build a complete duplex802.11bnode [9,10]. Expanded signal (for example, $802.11 \mathrm{n} 40 \mathrm{MHz}$ ) precludes full duplex WiFi transmit power. 


\section{Design of Full-duplex Wireless System}

In this section we describe a full-duplex wireless system, you only need two antennas and RF front-end design, no bandwidth limit, and automatically adjusts the self-interference cancellation in response to changing channel conditions. The design is based on a simple observation: any radio signal, you will always encounter boundary largest bandwidth constraints canceled by adjusting the phase. Cancel beyond this limit, the radio needs to be a signal that perfect inverse, which is transmitting a signal at all times completely negative signal. This fall and send a signal, in theory, can be completely eliminated from the combination of interference. All stations are required to adjust the phase of the signal is not inverted. Fortunately, there is a component, which is a balanced / unbalanced (unbalanced) transformer. Barron is a common component of RF and single-ended signal switch back and forth between --line signal has one thing in common - the polar opposite of the audio and video circuitry double signal - and the differential signal. For example, a single-ended signal in the coaxial cable. A differential signal transmission twisted pair (such as Ethernet), and vice verso, using a balun to take the signal as an input and output signal and its inverse.

The main point of this article is that people can use balun in a completely new way to get a signal from interfering with the inverse inverted signal to eliminate interference. Figure 2 shows a full-duplex radio antenna design, using a balun. Send a positive signal to the transmitting antenna. Cancellation of interference, radio interference combined with delays and negative signals from the received signal is a negative signal attenuation adjusted their matches.

We used to offset passive passive components to achieve high-precision variable attenuate and offset path delay. And in theory be completely eliminated offset, of course, there are some practical limitations. For example, the transmission signal attenuation and delay of experience in the air. Cancellation and get the perfect combination of the application must be in the same inverted signal attenuation and delay, it may be difficult to achieve in practice.

Learn inverted signal with the actual benefits and limitations Barron, compared to reversed phase and phase shift, we have strict control of RF test. Figure 3shows the experimental apparatus. A signal generator to generate a program of our center frequency $2.45 \mathrm{GHz} 240 \mathrm{MHz}$ wide band chirp. The signal passes through two lines. The first wire is an ideal self interference path representative antenna separation $20 \mathrm{~dB}$ attenuate. By eliminating the second wire path, including a variable actuator and the variable delay unit may be modified from the control path signal interference canceled match. The combination of these two signals flowing into a signal receiver. Variable delay line and attenuate manual tuning passives in this experiment allowed precision. We cancellation path than half a wavelength from interference path length through the analog antennas. RF Synthesizer plus two signal measurements canceled signal receiving side. Barron's settings, on the other hand, a balanced division of the transmitted signal, and use the same length of flexible wire him from interference and elimination pathway. In both cases, the passive delay lines and attenuation provides granular control and eliminate interference to match the path to maximize the elimination phase and amplitude.

To understand the real benefits and limitations of the reverse signal and the Ba Lun, we perform a rigorous control of the RF experiments as compared to the reverse phase and phase shift. A signal generator of our program generates a frequency $2.45 \mathrm{GHz}$ center broadband $240 \mathrm{MHz}$ chirp. The signal passes through two lines. The first wire is a kind of ideal self interference path which has a representative antenna $20 \mathrm{~dB}$ attenuate. The second wire is controlled by a variable attenuate and the variable delay unit can be controlled by a variable attenuate and a variable delay element can be controlled to match the self interference. The combination of these two signals flows into a signal receiver. In this experiment the variable delay line and attenuate manually tuned passive devices allow high accuracy.

We cancel the length of the path of half a wavelength ratio of the path to a self - interference path by simulating the antenna. Radio frequency synthesizer and the two signal of the receiving side to cancel the signal. The setting of the Ba Lun, on the other hand, uses a balanced splitting of the transmit signal, and uses the same length as the soft wire to self interference and eliminate the way. In these two cases, the passive delay lines and the attenuate provide a fine degree of control for the matched interference and eliminate the path to maximize the elimination of phase and amplitude. 
Figure 3 shows the results. Using phase shift signal canceled narrow bandwidth, but the cancellation of the broadband signal is very limited. Phase offset cancellation of $5 \mathrm{MHz}$ signal cancel $50 \mathrm{~dB}$, but only a100mhz signal is canceled. In contrast, based on the circuit it provides a good degree of canceling in a very wide bandwidth of balun. For example, Barron's cancellation will be provided and a signal $40 \mathrm{dbof}$ a a100mhz $5 \mathrm{MHz}$ of cancellation shall be canceled.

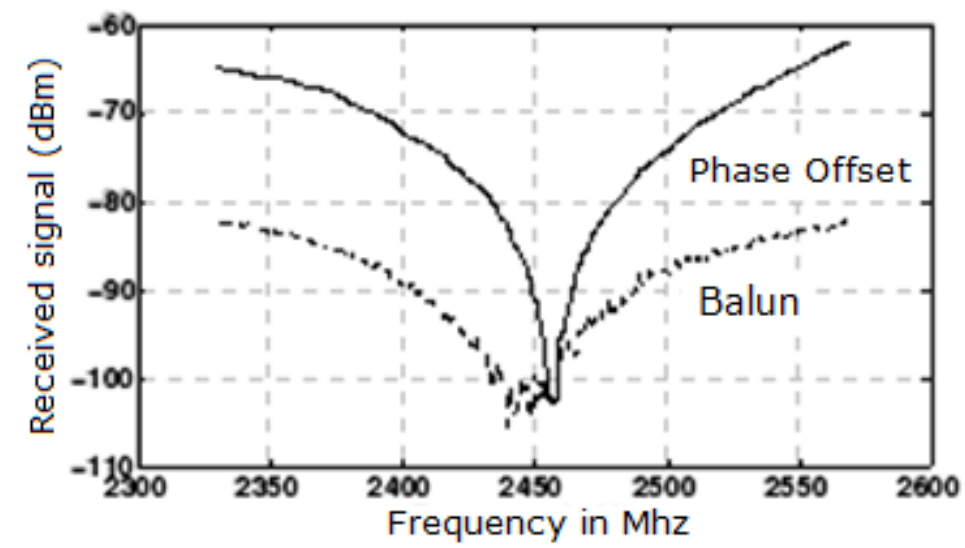

Figure 3: Self-interference phase shift signal cancellation.

Barron cancellation is not perfect, the entire band. The key reason is that the balun circuit is not flat frequency, i.e., bands of different portions having different amplitudes, inverted. Therefore, a single attenuation and delay factors inversion signal does not cancel the transmit signal perfect: This is a simple example of the real world of engineering tolerance limits theory. According to Figure 5, we can obtain a given signal bandwidth balance - unbalance and phase offset cancellation is best to cancel. Figure 6 shows the use of the best method to eliminate the various bands.

\section{Conclusion}

This paper presents the design of a new elimination technique for full duplex radio based on the so-called unbalanced transformer. Unlike existing designs, the balanced - unbalanced converter is able to cancel the signal work in broadband, high power, making it possible to build full duplex 802.11n devices. These devices can automatically adjust its cancellation circuit, so the operation in the dynamic environment. This paper describes the implementation of the full duplex prototype using a 802.11 - like OFDM link layer in real time operation. Using this prototype, it evaluates the full duplex MAC layer, which verifies the assumption that before the full duplex can prevent many hidden terminals and improve the fairness of WLAN.

\section{References}

[1] Rappaport T S, Shu S, Mayzus R. Millimeter wave mobile communications for 5G cellular: it will work. IEEEAccess , (2003),1: p335-349.

[2] Cadambe V R and Jafar S A. Degrees of freedom of wireless networks with relays, feedback, cooperation, and full duplex operation. IEEE Transactions on Information Theory,55(5): p. 2334-2344.

[3] Elsayed A, Eltawil A M, and Sabharwal A. Rate gain region and design tradeoffs for full-Duplex wireless communications . IEEE Transactions on Wireless Communications, (2013),12(7): p3556-3565.

[4] López-Valcarce R, Antonio-Rodríguez E, Mosquera C. An adaptive feedback canceller for full-duplex relays based on spectrum shaping. IEEE Journal on Selected Areas in Communications, (2012), 30(8): p1566-1577. 
[5] Napolitano A. Generalizations of Cyclostationary Signal Processing. Spectral Analysis and Applications. New York: Wiley, (2012): p45-121.

[6] Oppenheim A and Schafer R. Discrete Time Signal Processing. 3rd Ed., New Jersey: Prentice Hall Inc, (2009),p183-197.

[7] Choi J I, Jain M, Srinivasan K. Achieving single channel, full duplex wireless communication . MobiCom'10, New York, USA. (2010), p1-12.

[8] Radunovic B, Gunawardena D, Key P. Rethinking indoor wireless mesh design: low power, low frequency full-duplex. WIMESH, Boston, Massachusetts, (2010), p1-6.

[9] Jain M, Choi J, Kim T M. Practical, real-time, full duplex wireless. Mobile Computing and Networking, New York, USA, (2011), p301-312.

[10] Hong S, Mehlman J, and Katti S. Picasso. flexible RF and spectrum slicing. SIGCOMM'12, Helsinki, Finland,(2012), p13-17. 